\title{
TANDA: Transfer and Adapt Pre-Trained Transformer Models for Answer Sentence Selection
}

\author{
Siddhant Garg* \\ University of Wisconsin-Madison \\ Madison, WI, USA \\ sidgarg@cs.wisc.edu
}

\author{
Thuy Vu \\ Amazon Alexa \\ Manhattan Beach, CA, USA \\ thuyvu@amazon.com
}

\author{
Alessandro Moschitti \\ Amazon Alexa \\ Manhattan Beach, CA, USA \\ amosch@amazon.com
}

\begin{abstract}
We propose TANDA, an effective technique for fine-tuning pre-trained Transformer models for natural language tasks. Specifically, we first transfer a pre-trained model into a model for a general task by fine-tuning it with a large and highquality dataset. We then perform a second fine-tuning step to adapt the transferred model to the target domain. We demonstrate the benefits of our approach for answer sentence selection, which is a well-known inference task in Question Answering. We built a large scale dataset to enable the transfer step, exploiting the Natural Questions dataset. Our approach establishes the state of the art on two well-known benchmarks, WikiQA and TREC-QA, achieving the impressive MAP scores of $92 \%$ and $94.3 \%$, respectively, which largely outperform the the highest scores of $83.4 \%$ and $87.5 \%$ of previous work. We empirically show that TANDA generates more stable and robust models reducing the effort required for selecting optimal hyper-parameters. Additionally, we show that the transfer step of TANDA makes the adaptation step more robust to noise. This enables a more effective use of noisy datasets for fine-tuning. Finally, we also confirm the positive impact of TANDA in an industrial setting, using domain specific datasets subject to different types of noise.
\end{abstract}

\section{Introduction}

In recent years, virtual assistants have become a central asset for technological companies. This has increased the interest of AI researchers in studying and developing conversational agents, some popular examples being Google Home, Siri and Alexa. This has renewed the research interest in Question Answering (QA) and, in particular, in two main tasks: (i) answer sentence selection (AS2), which, given a question and a set of answer sentence candidates, consists in selecting sentences (e.g., retrieved by a search engine) correctly answering the question; and (ii) machine reading (MR) (Chen et al. 2017) or reading comprehension, which, given a question and a reference text, consists in finding a text span answering it. Even though the latter is gaining more and more popularity, AS2 is more relevant to a production scenario

\footnotetext{
${ }^{*}$ Work done while the author was an intern at Amazon Alexa. Copyright (c) 2020, Association for the Advancement of Artificial Intelligence (www.aaai.org). All rights reserved.
}

since, a combination of a search engine and an AS2 model already implements an initial QA system.

The AS2 task was originally defined in the TREC competition (Wang, Smith, and Mitamura 2007). With the advent of neural models, it has had significant contributions from techniques such as (He and Lin 2016; Yang et al. 2018; Wang and Jiang 2016).

Recently, approaches for training neural language models, e.g., ELMO (Peters et al. 2018), GPT (Radford et al. 2018), BERT (Devlin et al. 2018), RoBERTa (Liu et al. 2019), XLNet (Dai et al. 2019) have led to major advancements in several NLP subfields. These methods capture dependencies between words and their compounds by pre-training neural networks on large amounts of data. Interestingly, the resulting models can be easily applied to solve different NLP applications by just fine-tuning them on the training data of the target tasks. For example, the Transformer (Vaswani et al. 2017) can be pre-trained on a large amount of data obtaining a powerful language model, which can then be specialized for solving specific NLP tasks by just adding new layers and training them on the target data. Although the approach is simple, the procedure for fine-tuning a Transformer-based model is not completely understood, and can result in high accuracy variance, with a possible on-off behavior, e.g., models may always predict a single label. As a consequence, researchers need to invest a considerable effort in selecting suitable parameters, with no theory or a well-assessed best practice helping them. Such problem also affects QA, and, in particular, AS2 since no large and and accurate dataset has been developed for it.

In this paper, we study the use of Transformer-based models for AS2 and provide effective solutions to tackle the data scarceness problem for AS2 and the instability of the finetuning step. In detail, the contributions of our papers are:

- We improve stability of Transformer models by adding an intermediate fine-tuning step, which aims at specializing them to the target task (AS2), i.e., this step transfers a pretrained language model to a model for the target task.

- We show that the transferred model can be effectively adapted to the target domain with a subsequent finetuning step, even when using target data of small size.

- Our Transfer and Adapt (TANDA) approach makes fine- 
tuning: (i) easier and more stable, without the need of cherry picking parameters; and (ii) robust to noise, i.e., noisy data from the target domain can be utilized to train an accurate model.

- We built ASNQ, a dataset for AS2, by transforming the recently released Natural Questions (NQ) corpus (Kwiatkowski et al. 2019) from MR to AS2 task. This was essential as our transfer step requires a large and accurate dataset. ASNQ is an important contribution of our work to the research community. ${ }^{1}$

- Finally, the generality of our approach and empirical investigation suggest that our TANDA findings also apply to other NLP tasks, especially, textual inference, although empirical analysis is essential to confirm these claims.

We evaluated TANDA on two well-known academic benchmarks, i.e., TREC-QA (Wang, Smith, and Mitamura 2007) and WikiQA (Yang, Yih, and Meek 2015), as well as three different industrial datasets, where questions are derived from Alexa traffic and candidate sentences are selected from web data. The results show that:

- TANDA improves the stability of Transformer models. In the adapt step, the model accuracy throughout different epochs show a smooth and convex behavior, which is ideal for estimating optimal parameters.

- We improved the state of the art in AS2 by just applying BERT and RoBERTa to AS2 and further improved it by almost 10 absolute percent points in MAP with TANDA.

- TANDA achieves much higher accuracy than traditional fine-tuning, especially in case of noise data. For example, the drop in performance is up to one order of magnitude lower with TANDA, i.e., $2.5 \%$, when we inject $20 \%$ of noise in the WikiQA and TREC-QA datasets.

- Our experiments with real-world datasets built from Alexa traffic data confirm all our above findings. Specifically, we observe the same robustness to noise, which, in this case, is generated by real sources.

The rest of the paper is structured as follows: we describe related work in Section 2, details of our TANDA approach in Section 3, present the new AS2 dataset in Section 4. The experimental results on academic benchmarks, including experiments with noise data, are reported in Section 5, while the results on real-world data are illustrated in Section 6. Finally, we derive conclusions in Section 7.

\section{Related Work}

Recent AS2 models are based on Deep Neural Networks (DNNs), which learn distributed representations of the input data and are trained to apply a series of non-linear transformations to the input question and answer, represented as compositions of word or character embeddings. DNN architectures learn answer sentence-relevant patterns using intrapair similarities as well as cross-pair, question-to-question and answer-to-answer similarities, when modeling the input

\footnotetext{
${ }^{1}$ The ASNQ dataset and trained models can be accessed at https://github.com/alexa/wqa_tanda
}

texts. For example, the CNN network by Severyn and Moschitti has two separate embedding layers for the question and answer, which are followed by the respective convolution layers. The output of the latter is concatenated and then passed through the final fully-connected joint layer. They also added embeddings encoding relational links between matching words (Severyn and Moschitti 2016): a sort of hardcoded attention, which highly increases accuracy.

More recent papers (Shen, Yang, and Deng 2017; Tran et al. 2018; Tay, Tuan, and Hui 2018) also propose a technique of inter-weighted alignment networks for this task. While others (Wang and Jiang 2016; Bian et al. 2017; Yoon et al. 2019) use a compare aggregate architecture, which also exploits an attention mechanism over the question and answer sentence embeddings. Tayyar Madabushi, Lee, and Barnden (2018) propose a method that integrates fine-grained Question Classification with a Deep Learning model designed for AS2.

Previous work (Wang, Smith, and Mitamura 2007) was carried out on relatively small datasets compared to other NLP tasks, such as machine translation, e.g., the WMT15 English-Czech dataset (Luong and Manning 2016) contains 15.8 million sentence pairs. This further motivates our work of creating a new large scale AS2 dataset, ASNQ, which is two orders of magnitudes larger than datasets such as TRECQA (Wang, Smith, and Mitamura 2007).

ASNQ is based on the NQ dataset, which is a corpus designed to (i) study MR tasks, and (ii) solve several problems of previous corpora such as SQuAD (Rajpurkar et al. 2016). MR models, e.g., (Seo et al. 2016), are different from those used for AS2 and beyond the purpose of our paper. It is still worthwhile to mention that Min, Seo, and Hajishirzi (2017) explored transfer learning for the BiDAF model for MR.

Very recent works (Devlin et al. 2018; Yang et al. 2019) use pre-trained Transformer models for MR. In this context, TANDA and ASNQ may provide alternative research directions, e.g., a more stable model that can then be fined tuned to the MR task.

Wang et al. (2019) report some marginal improvement for the task of text classification by fixing weights of Transformer models derived by BERT, when training the classification layer. Sun et al. (2019) carried out additional pretraining of BERT-derived models on the target dataset. They also used different learning rates for different model layers.

\section{TANDA: Transfer and Adapt}

We propose to train Transformer models for the AS2 by applying a two-step fine-tuning, called Transfer AND Adapt (TANDA). The first step transfers the language model of the Transformer to the AS2 task; and the second fine-tuning step adapts the obtained model to the specific target domain, i.e., specific types of questions and answers. We first provide a background on AS2 and Transformer models, and, then explain our approach in more detail.

\subsection{AS2 task and model definition}

AS2 can be defined as follows: given a question $q$ and a set of answer sentence candidates $S=\left\{s_{1}, . ., s_{n}\right\}$, select a sentence $s_{k}$ that correctly answers $q$. We can model the task as a 


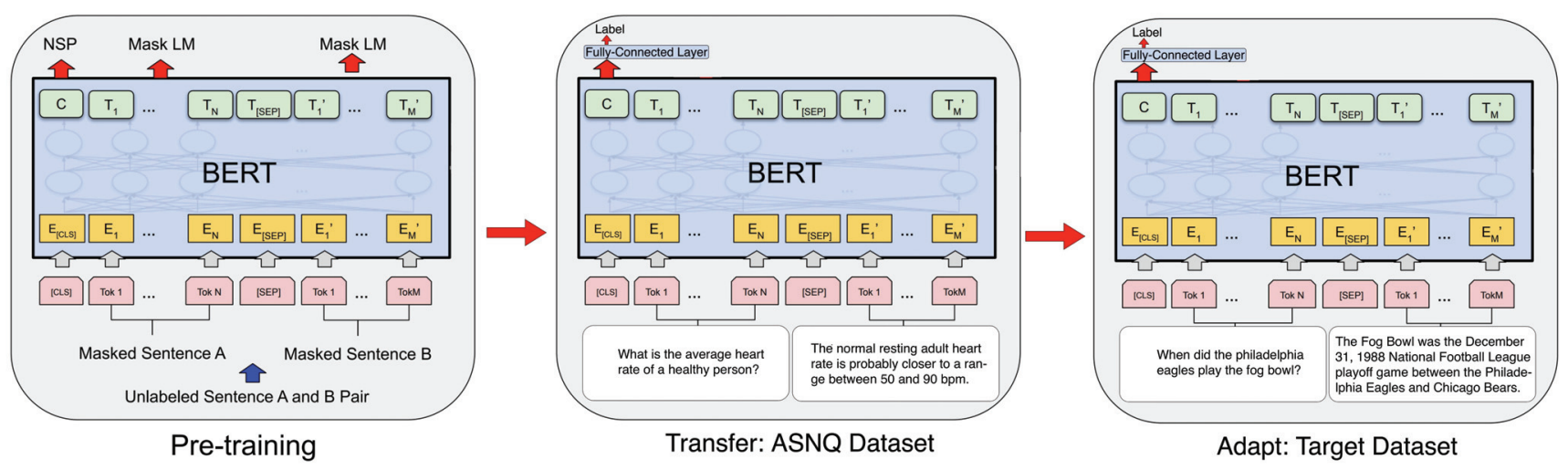

Figure 1: Transfer and Adapt for Answer Sentence Selection, applied to BERT

function $r: Q \times \mathcal{P}(S) \rightarrow S$, defined as $r(q, S)=s_{k}$, where $k=\operatorname{argmax}_{i} p\left(q, s_{i}\right)$ and $p\left(q, s_{i}\right)$ is the probability of correctness of $s_{i}$. We estimate $p\left(q, s_{i}\right)$ using neural networks, in particular, Transformer models, as explained below.

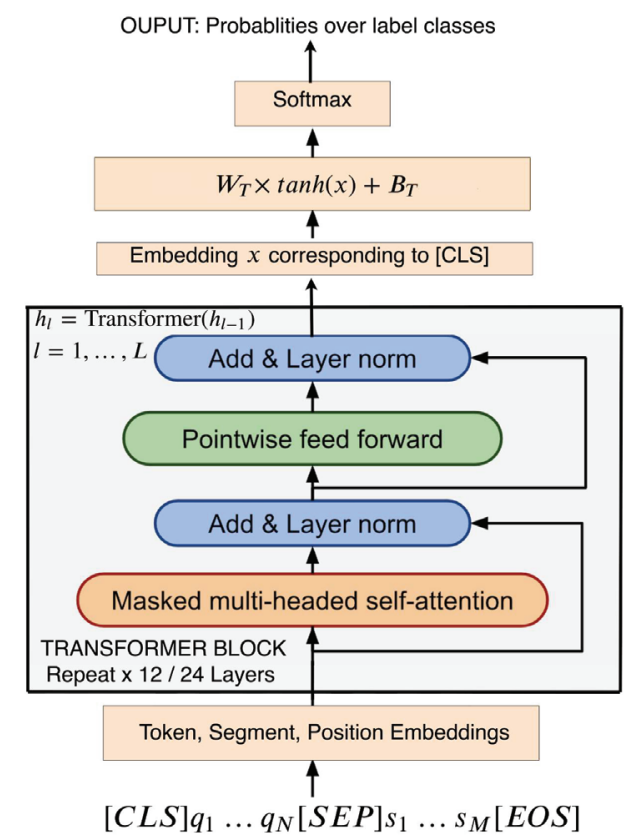

Figure 2: Transformer architecture with on top a linear classifier for fine-tuning on AS2. Here [CLS] $\operatorname{Tok}_{1}^{1}, \ldots, \operatorname{Tok}_{N}^{1}$ [SEP] $\operatorname{Tok}_{1}^{2}, \ldots, \operatorname{Tok}_{M}^{2}[\mathrm{EOS}]$ is the input to the model.

\subsection{Transformer models for AS2}

Transformer-based models are neural networks designed to capture dependencies between words, i.e., their interdependent contexts. Fig. 2 shows the standard architecture for a text pair classification task. The input consists of two pieces of text, $\operatorname{Tok}_{1}^{1}, \ldots, \mathrm{Tok}_{N}^{1}$ and $\mathrm{Tok}_{1}^{2}, \ldots, \mathrm{Tok}_{M}^{2}$ delimited by three tags, [CLS], [SEP] and [EOS] (beginning, separator and end of sentence). The input is encoded as embeddings based on tokens, segments and their positions. These are fed as input to several blocks (up to 24) containing layers for multi-head attention, normalization and feed forward processing. The result of this transformation is an embedding, $\mathbf{x}$, representing the text pair, which models the dependencies between words and segments of the two sentences. For a downstream task, $\mathbf{x}$ is fed (after applying a non linearity function) to a fully connected layer having weights: $W_{T}$ and $B_{T}$. The output layer can be used to implement the task function. For example, a softmax can be used to model the probability of a text pair classification, as described by the equation: $\hat{y}=\operatorname{softmax}\left(W_{T} \times \tanh (x)+B_{T}\right)$.

Theoretically, this model can be trained using the log cross-entropy loss: $\mathcal{L}=-\sum_{l \in\{0,1\}} y_{l} \times \log \left(\hat{y}_{l}\right)$ on pairs of text. In practice, a large amount of supervised data will be required for this step of training. The important contribution by Devlin et al., (2018) was to pre-train the language model, i.e., the sentence pair representation, on using surrogate tasks such as masked language modeling and next sentence prediction.

The left block in Fig. 1 illustrates the pre-training step of a Transformer model, highlighting that some words in the pair are masked. This way the model learns to generalize the input while providing the same output. The default transfer approach, defined in previous work, fine-tunes the Transformer model to the target task and domain (in one shot). For AS2, the training data comprises of question and sentence pairs with positive or negative labels according to the test: the sentence correctly answers the question or not. This fine-tuning is rather critical as the initial task learned during the pre-training stage is very different from AS2.

When only small target data is available, the transfer process from the language model to AS2 task is unstable. We conjecture that a large number of examples are required to fine-tune the large number of Transformer parameters on the new task. An evidence of this is the on-off effect, that is, the fine-tuned model always predicts a single label for all examples. More in general, the model accuracy is unstable showing a large variance over different fine-tuning attempts.

We explain this behavior considering the quality and quantity of the training data required for the transfer step 
(from the pre-trained model to AS2). More precisely, a small number of data examples prevents an effective convergence to the task, while noisy data leads to incorrect convergence. Thus, we propose to divide the fine-tuning process in two steps: transfer to the task and then adapt to the target domain (TANDA). This is advantageous over a single fine-tuning step, since the latter would require either (i) the availability of a large dataset for the target domain, which is undesirable due to higher difficulty and cost of collection of domain specific data over general data; or (ii) merging the general and domain specific data in a single training step, which is not optimal since the the model needs to be specialized only to the target data. In principle when using a combination of general and domain specific data, instance weighting can be used by giving more importance to the target data. However, finding the right weights is complex as neural models do not converge to a global optimum: thereby leading to very different outcomes for different weights.

\subsection{TAndA}

The two steps of our approach are depicted in the center and right blocks of Fig. 1. We apply a standard fine-tuning step using a large scale general purpose dataset for AS2. This step is supposed to transfer the Transformer language model to the AS2 task. The resulting model will not perform optimally on the data of the target domain due to the specificity of the latter. We thus apply a second fine-tuning step to adapt the classifier to the target AS2 domain. For example, in the transfer step, we may have general questions such as, What is the average heart rate of a healthy person while, in the adapt step, the target domain, e.g., sport news, may contain specific questions such as: When did the Philadelphia eagles play the fog bowl?

Using different training steps on the target data to improve performance is a rather intuitive approach. In this paper, we highlight the role of the transfer step, which (i) greatly reduces the amount of data required in the adaptation step; and (ii) stabilizes the model, making it robust to noise. We empirically demonstrate both claims in our experiments.

\section{Answer-Sentence Natural Questions}

We needed an accurate, general and large AS2 corpus to validate the benefits of TANDA. Since existing AS2 datasets are small in size, we built a new AS2 dataset called Answer Sentence Natural Questions (ASNQ) derived from the recently released Google Natural Questions (NQ) dataset (Kwiatkowski et al. 2019).

NQ is a large scale dataset intended for the MR task, where each question is associated with a Wikipedia page. For each question, a long paragraph (long_answer) that contains the answer is extracted from the reference page. Each long_answer may contain phrases annotated as short_answer. A long_answer can contain multiple sentences, thus NQ is not directly applicable for AS2.

For each question in ASNQ, the positive candidate answers are those sentences that occur in the long answer paragraphs in NQ and contain annotated short answers. The remaining sentences from the document are labeled as nega-
Question: What is the highest mountain in france called ?

Mont Blanc (pronounced ( mõ blã ); Italian: Monte Bianco ('monte 'bjanko)) meaning "White Mountain", is the highest mountain in the Alns and the hiahest in Furone west of Russia's Caucasus neaks It rises $4,808 \mathrm{~m}(15,774 \mathrm{ft})$ above sea level and is ranked 11th in the world in topographic prominence. The mountain stands in a range called the Graian Alps, between the reaions of Aosta Vallev. Italv. and Savoie and Haute-Savoie. France. The location of the summit is on the watershed line between the valleys of Ferret and Venv in Italv and the vallevs of Montioie. and Arve in France. The Mont Blanc massif is popular for hikino akiina and snowboardina. The three towns and their communes which surround Mont Blanc are Courmayeur in Aosta Valley, Italv: and Saint-Gervais-les-Bains and Chamonix in Haute-Savoie. France. A cable car ascends and crosses the mountain range from Courmayeur to Chamonix, throuah the Col du Géant.

Long Answer Mont Blanc: Annotated Short Answer Mont Blanc : Non-Annotated Short Answer Label 4

Figure 3: An example of data instance conversion from NQ to ASNQ.

\begin{tabular}{|c|c|c|c|c|}
\hline Label & $\mathrm{S} \in \mathrm{LA}$ & $\mathrm{SA} \in \mathrm{S}$ & \# Train & \# Dev \\
\hline 1 & No & No & $19,446,120$ & 870,404 \\
\hline 2 & No & Yes & 428,122 & 25,814 \\
\hline 3 & Yes & No & 442,140 & 29,558 \\
\hline 4 & Yes & Yes & 61,186 & 4,286 \\
\hline
\end{tabular}

Table 1: Label description for ASNQ. Here S, LA, SA refer to answer sentence, long answer passage and short answer phrase respectively.

tive for the target question. The negative examples can be of the following types:

1. Sentences from the document that are in the long_answer but do not contain the annotated short answers. It is possible that these sentences might contain the short_answer.

2. Sentences from the document that are not in the long_answer but contain the short_answer string, that is, such occurrence is purely accidental.

3. Sentences from the document that are neither in the long_answer nor contain the short_answer.

The generation of negative examples is particularly impactful to the robustness of the model in identifying the best answer out of the similar but incorrect ones. ASNQ has four labels that describe possible confusing levels of a sentence candidate. We apply the same processing both to training and development sets of NQ. An example is shown in Fig. 3, while the ASNQ statistics are reported in Table 1.

ASNQ contains 57,242 distinct questions in the training set and 2,672 distinct questions in the dev. set, which is an order of magnitude larger than most public AS2 datasets. For the transfer step in TANDA, we use ASNQ sentence pairs with labels 1, 2 and 3 as negatives and Label 4 as positives.

\section{Experiments on Standard Benchmarks}

We provide empirical evidence on the benefits of using TANDA on two commonly used benchmarks for AS2: 


\begin{tabular}{|l|l|l|l|l|l|l|}
\hline \multirow{2}{*}{ Mode } & \multicolumn{3}{|l|}{ Train } & \multicolumn{2}{l|}{ Dev } & \multicolumn{2}{l|}{ Test } \\
\cline { 2 - 7 } & Q & A & Q & A & Q & A \\
\hline raw & 2118 & 20360 & 296 & 2733 & 633 & 6165 \\
\hline no all- & 873 & 8672 & 126 & 1130 & 243 & 2351 \\
\hline Clean & 857 & 8651 & 121 & 1126 & 237 & 2341 \\
\hline
\end{tabular}

Table 2: WikiQA dataset statistics

WikiQA and TREC-QA, which enable a direct comparison with previous work.

\subsection{Academic Datasets}

WikiQA (Yang, Yih, and Meek 2015) has some questions with no correct answer sentence (all-) or with only correct answer sentences (all+). Table 2 reports the corpus statistics of the versions: raw (as distributed), without all- questions, and without both all- and all + questions (Clean). We follow the most used setting: training with the no all- mode and then answer candidate sentences per question in testing with the Clean mode.

TREC-QA is another popular benchmark (Wang, Smith, and Mitamura 2007) for AS2. We removed questions without answers, or with only correct or only incorrect answer sentence candidates, from the dev. and test sets. This resulted in 1, 229, 65 and 68 questions, and 53, 417, 1, 117 and 1, 442 question-answer pairs for training, dev. and test sets, respectively. This setting refers to the Clean setting (Shen, Yang, and Deng 2017), which is a TREC-QA standard.

QNLI, namely, Question Natural Language Inference is a dataset (Wang et al. 2018) derived from the Stanford Question Answering Dataset (SQuAD) (Rajpurkar et al. 2016) by converting question-paragraph pairs into sentence pairs, resulting in dataset with $105 \mathrm{k}$ question-answer pairs for training and $5.4 \mathrm{k}$ pairs in the dev. data. We carry out experiments of using QNLI for the transfer step in TANDA and compare it with previous methods (Yoon et al. 2019), which are based on QNLI for sequential fine-tuning of nonTransformer models for AS2.

\subsection{Training and testing details}

Metrics We measure system accuracy with Mean Average Precision (MAP) and Mean Reciprocal Recall (MRR) evaluated on the test set, using the entire set of candidates for each questions (this varies according to the different datasets).

Models We use the pre-trained BERT-Base (12 layer), BERT-Large (24 layer), RoBERTa-Base (12 layer) and RoBERTa-Large-MNLI (24 layer) models, which were released as checkpoints for use in downstream tasks.

Training We adopt Adam optimizer (Kingma and $\mathrm{Ba}$ 2014) with a learning rate of 2e-5 for the transfer step on the ASNQ dataset and a learning rate of 1e-6 for the adapt step on the target dataset. We apply early stopping on the dev. set of the target corpus for both steps based on the highest MAP score. We set the max number of epochs equal to 3 and 9 for adapt and transfer steps, respectively. We set the maximum sequence length for BERT/RoBERTa to 128 tokens.

\begin{tabular}{|l|c|c|}
\hline Model & MAP & MRR \\
\hline Comp-Agg + LM + LC & 0.764 & 0.784 \\
\hline Comp-Agg + LM + LC+ TL(QNLI) & 0.834 & 0.848 \\
\hline \hline BERT-B FT WikiQA & 0.813 & 0.828 \\
\hline BERT-B FT ASNQ & 0.884 & 0.898 \\
\hline BERT-B TANDA (ASNQ $\rightarrow$ WikiQA ) & 0.893 & 0.903 \\
\hline \hline BERT-L FT WikiQA & 0.836 & 0.853 \\
\hline BERT-L FT ASNQ & 0.892 & 0.904 \\
\hline BERT-L TANDA (ASNQ $\rightarrow$ WikiQA) & 0.904 & 0.912 \\
\hline \hline RoBERTa-B FT ASNQ & 0.882 & 0.894 \\
\hline RoBERTa-B TANDA (ASNQ $\rightarrow$ WikiQA) & 0.889 & 0.901 \\
\hline RoBERTa-L FT ASNQ & 0.910 & 0.919 \\
\hline RoBERTa-L TANDA (ASNQ $\rightarrow$ WikiQA ) & $\mathbf{0 . 9 2 0}$ & $\mathbf{0 . 9 3 3}$ \\
\hline
\end{tabular}

Table 3: Performance of different models on WikiQA dataset. Here Comp-Agg + LM + LC refers to a CompareAggregate model with Language Modeling and Latent Clustering as proposed by Yoon et al. (2019). TL(QNLI) refers to Transfer Learning from the QNLI corpus. L and B stand for Large and Base, respectively.

Parameter Tuning We selected learning rates for Adam optimizer in the transfer and adapt steps as follows: (i) We tested a reasonable set of values for the transfer and adapt steps, identifying two promising values, $1 e-6$ and $2 e-5$, for the former, and five values $\{1 e-6,2 e-6,5 e-6,1 e-$ $5,2 e-5\}$ for the latter. These are within the range of typical learning rates for the Adam optimizer. (ii) We tested the ten combinations for the TANDA approach and we selected the value pair, $(2 e-5,1 e-6)$, for the transfer and adapt step respectively, which optimizes the MAP on the dev. set of the target dataset. As TANDA makes fine-tuning stable, we ended up selecting the same parameter values for all tested datasets. It should be noted that the optimality of a larger learning rate for the first step and a smaller learning rate for the second step supports our claim of considering the second step of TANDA as domain adaptation process. For the experiments with one fine-tuning step (baseline), we again chose either $1 e-6$ or $2 e-5$, i.e, following the common practice of BERT fine-tuning.

\subsection{Main Results}

WikiQA Table 3 reports the MAP and MRR of different pre-trained Transformer models for two methods: standard fine-tuning (FT) and TANDA. The latter takes two arguments that we indicate as transfer dataset $\rightarrow$ adapt dataset. The table shows that:

- BERT-Large and BERT-Base with standard fine-tuning on WikiQA match the current state of the art by Yoon et al. (2019). ${ }^{2}$ The latter uses the compare aggregate model with latent clustering, ELMO embeddings, and transfer learning from the QNLI corpus.

- TANDA provides a large improvement over the state of the art, which has been regularly contributed to by hundreds of researchers.

\footnotetext{
${ }^{2}$ https://aclweb.org/aclwiki/Question_Answering_(State_of the_art)
} 


\begin{tabular}{|l|c|c|}
\hline Model & MAP & MRR \\
\hline Comp-Agg + LM + LC & 0.868 & 0.928 \\
\hline Comp-Agg + LM + LC + TL(QNLI) & 0.875 & 0.940 \\
\hline \hline BERT-B FT TREC-QA & 0.857 & 0.937 \\
\hline BERT-B FT ASNQ & 0.823 & 0.872 \\
\hline BERT-B TANDA (ASNQ $\rightarrow$ TREC-QA) & 0.912 & 0.951 \\
\hline \hline BERT-L FT TREC-QA & 0.904 & 0.946 \\
\hline BERT-L FT ASNQ & 0.824 & 0.872 \\
\hline BERT-L TANDA (ASNQ $\rightarrow$ TREC-QA ) & 0.912 & 0.967 \\
\hline \hline RoBERTa-B FT ASNQ & 0.849 & 0.907 \\
\hline RoBERTa-B TANDA (ASNQ $\rightarrow$ TREC-QA ) & 0.914 & 0.952 \\
\hline RoBERTa-L FT ASNQ & 0.880 & 0.928 \\
\hline RoBERTa-L TANDA (ASNQ $\rightarrow$ TREC-QA) & $\mathbf{0 . 9 4 3}$ & $\mathbf{0 . 9 7 4}$ \\
\hline
\end{tabular}

Table 4: Performance of different models on TREC-QA dataset. Here Comp-Agg + LM + LC refers to a CompareAggregate model with Language Modeling and Latent Clustering as proposed in (Yoon et al. 2019). TL(QNLI) refers to Transfer Learning from the QNLI corpus. L and B stand for Large and Base, respectively.

- RoBERTa-Large TANDA using ASNQ $\rightarrow$ WikiQA establish an impressive new state of the art for AS2 on WikiQA of 0.920 and 0.933 in MAP and MRR, respectively.

- Finally, we note that the standard fine-tuning on ASNQ already outperforms the previous state of the art. This is mainly due to the fact that as ASNQ and WikiQA are both based on answers from Wikipedia.

The next section confirms the results above on TREC-QA.

TREC-QA Table 4 reports the results of our experiments with TREC-QA. We note that:

- RoBERTa-Large TANDA with ASNQ $\rightarrow$ TREC-QA again establishes an impressive performance of 0.943 in MAP and 0.974 in MRR, outperforming the previous state of the art by Yoon et al. (2019).

- Both BERT-Base and Large fine purely tuned on the TREC-QA corpus can surpass the previous state of the art, probably because the size of TREC-QA training corpus is larger than that of WikiQA.

- TANDA improves all the models: BERT-Base, RoBERTaBase, BERT-Large and RoBERTa-Large, outperforming the previous state of the art with all of them.

- Finally, the model obtained with FT on just ASNQ produces the expected results: it performs much lower than any TANDA model and also lower than FT on just TREC-QA since the target domain of TREC questions is significantly different from that of ASNQ.

We also tried FT on the merged ASNQ and TREC-QA dataset to show that the sequential FT, i.e., TANDA, improves over this. BERT-Base model fine-tuned on ASNQ $U$ TREC-QA achieves a MAP and MRR of 0.898 and 0.929, respectively. These are significantly lower than 0.912 MAP and 0.951 MRR, obtained with TANDA. We also stress the other important benefits of TANDA: (i) it enables modularity, thereby avoiding to retrain on the large ASNQ data (FT on any target dataset can start from the model transferred

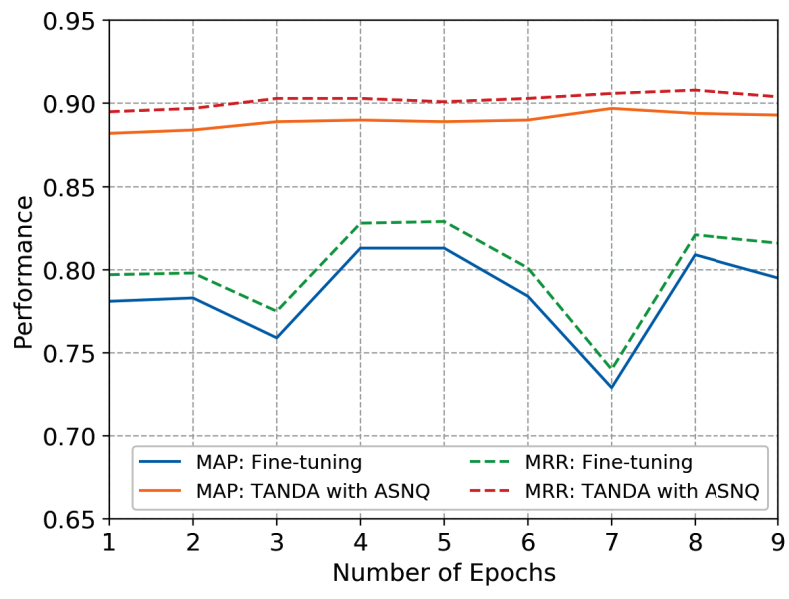

Figure 4: MAP and MRR on the WikiQA-Test-data varying with number of fine-tuning epochs on the WikiQA-Traindata for simple FT and TANDA.

with ASNQ); and (ii) it has a higher training efficiency (target datasets are much smaller than ASNQ).

\subsection{Properties of TANDA}

Stability of TANDA Systematic and effective fine-tuning of Transformer models is still an open problem. There is no theory or even a well-assessed best practice suggesting the optimal number of epochs to be used for fine-tuning. We claim that TANDA can robustly transfer language models to the target task and this produces more stable models. For stability, we mean a low variance of the model accuracy (i) between two consecutive training epochs, and (ii) between two pairs of models that have close accuracy on the development set. For example, BERT FT has a high variance in accuracy with the number of epochs, leading to some extreme cases of an on-off behavior, i.e, the classifier may only predict negative labels for target task (due to unbalanced datasets).

To test our hypothesis, we compared TANDA with standard FT by varying the number of training epochs for the adaptation step (here we do not use early stopping for TANDA). Figure 4 shows the plots of MAP and MRR scores with BERT-Base on WikiQA test set with different number of training epochs on the train set. As expected FT has a high variance while TANDA shows a small variance. A direct consequence of this better behavior is the higher probability to select an optimal epoch parameter on the dev. set.

Robustness to Noise in WikiQA and TREC-QA Better model stability also means robustness to noise. We empirically studied this conjecture by artificially injecting noise in the training sets of WikiQA and TREC-QA, by randomly sampling questions-answer pairs from the training set and switching their label values. We chose random samples of $10 \%$ and $20 \%$ of the training data, generating 867 and 1734 noisy labels, on WikiQA, respectively, and 5341 and 10683 noisy labels, on TREC-QA, respectively. We used the same Clean data setting to directly compare with the original performance. Table 5 shows the MAP and MRR of BERT- 


\begin{tabular}{|c|c|c|c|c|c|c|c|c|}
\hline \multirow{2}{*}{ BERT-base } & \multicolumn{4}{|c|}{ WikiQA } & \multicolumn{4}{c|}{ TREC-QA } \\
\cline { 2 - 9 } & MAP & $\%$ Drop & MRR & $\%$ Drop & MAP & \% Drop & MRR & $\%$ Drop \\
\hline No noise Fine-tuning & 0.813 & - & 0.828 & - & 0.857 & - & 0.937 & - \\
\hline $10 \%$ noise Fine-tuning & 0.775 & $4.67 \%$ & 0.793 & $4.22 \%$ & 0.826 & $3.62 \%$ & 0.902 & $3.73 \%$ \\
\hline $20 \%$ noise Fine-tuning & 0.629 & $\mathbf{2 2 . 6 3} \%$ & 0.645 & $22.10 \%$ & 0.738 & $\mathbf{1 3 . 8 8} \%$ & 0.843 & $10.03 \%$ \\
\hline No noise TANDA (ASNQ $\rightarrow *$ ) & 0.893 & - & 0.903 & - & 0.912 & - & 0.951 & - \\
\hline $10 \%$ noise TANDA (ASNQ $\rightarrow *$ ) & 0.876 & $1.90 \%$ & 0.889 & $1.55 \%$ & 0.896 & $1.75 \%$ & 0.941 & $1.05 \%$ \\
\hline $20 \%$ noise TANDA (ASNQ $\rightarrow *$ ) & 0.870 & $\mathbf{2 . 5 7} \%$ & 0.886 & $1.88 \%$ & 0.891 & $\mathbf{2 . 3 0} \%$ & 0.937 & $1.47 \%$ \\
\hline
\end{tabular}

Table 5: Model accuracy when noise is injected into WikiQA and TREC-QA datasets. $*$ indicates the target dataset for the second step of fine-tuning (adapt step).

Base using FT and TANDA, also indicating the drop percentage $(\%)$ in accuracy due to the injection of noise. We note that standard FT is highly affected by noisy data, e.g., on WikiQA the accuracy decreases by $22.63 \%$, when $20 \%$ of noise is injected. Very interestingly, the models using TANDA are affected an order of magnitude less, i.e., just $2.57 \%$. A similar trend can be observed aslo for the results on TREC-QA.

\begin{tabular}{|l|l|l|l|l|}
\hline \multirow{2}{*}{ Model } & \multicolumn{2}{|l|}{ WikiQA } & \multicolumn{2}{l|}{ TREC-QA } \\
\cline { 2 - 5 } & MAP & MRR & MAP & MRR \\
\hline Neg: 1 Pos: 4 & 0.870 & 0.880 & 0.808 & 0.847 \\
\hline Neg: 2 Pos: 4 & 0.751 & 0.763 & 0.662 & 0.751 \\
\hline Neg: 3 Pos: 4 & 0.881 & 0.895 & 0.821 & 0.869 \\
\hline Neg: 2,3 Pos: 4 & 0.883 & 0.898 & 0.823 & 0.871 \\
\hline Neg: 1,2,3 Pos: 4 & 0.884 & 0.898 & 0.823 & 0.872 \\
\hline
\end{tabular}

Table 6: Impact of different labels of ASNQ on fine-tuning BERT for answer sentence selection. Neg and Pos refers to question-answer (QA) pairs of that particular label being chosen for fine-tuning.

\subsection{Insights on ASNQ}

Ablation studies Fig. 3 shows that we generated different types of negative examples for the AS2 task, namely, labels 1,2 and 3. We carried out experiments by fine-tuning BERTBase on ASNQ with specific label categories assigned to the negative class. Table 6 shows the results: Label 3 is the most effective negative type of the three, i.e., the models only using Label 3 as negative class are just subject to a marginal drop in performance with respect to the model using all labels. Labels 2 and 3 provide the same accuracy than the three labels as the negative class.

\begin{tabular}{|l|l|l|l|l|}
\hline \multirow{2}{*}{ BERT-Base } & \multicolumn{2}{|l|}{ WikiQA } & \multicolumn{2}{l|}{ TREC-QA } \\
\cline { 2 - 5 } & MAP & MRR & MAP & MRR \\
\hline FT QNLI & 0.760 & 0.766 & 0.820 & 0.890 \\
\hline FT ASNQ & 0.884 & 0.898 & 0.823 & 0.872 \\
\hline TANDA (QNLI $\rightarrow$ ) & 0.832 & 0.852 & 0.863 & 0.906 \\
\hline TANDA (ASNQ $\rightarrow$ ) & 0.893 & 0.903 & 0.912 & 0.951 \\
\hline
\end{tabular}

Table 7: Comparison of TANDA with ASNQ and QNLI
ASNQ vs. QNLI Another way to empirically evaluate the impact of ASNQ is to compare it with other similar datasets, e.g., QNLI, observing the performance of the latter when used for a simple FT or in TANDA. Table 7 shows that both FT and TANDA using ASNQ provide significantly better performance than QNLI on the WikiQA dataset.

On TREC-QA dataset the results show that (i) FT on QNLI performs better than ASNQ but (ii) when TANDA uses ASNQ as transfer step, the models can better adapt to TREC-QA data than when using QNLI for the same transfer type. On one hand, this confirms the claim about the high quality of ASNQ. It is a more general and accurate AS2 dataset and is better suited for transferring the Transformer language model. On the other hand, it provides some evidence that the transfer step is very important and is not just a way for initializing weights of the adaptation step.

\begin{tabular}{|c|c|c|c|c|}
\hline Dataset & Questions & QA Pairs & Pos. & Neg. \\
\hline Sample 1 & 435 & 21,530 & 19,598 & 1,932 \\
\hline Sample 2 & 441 & 44,593 & 40,136 & 4,457 \\
\hline Sample 3 & 452 & 45,300 & 42,131 & 3,169 \\
\hline
\end{tabular}

Table 8: Statistics of samples 1, 2 and 3 (accurate test sets)

\section{Experiments on data from Alexa}

To show that our results generalize well, we tested our models using four different AS2 datasets created with questions sampled from the customers' interaction with Alexa Virtual Assistant.

\subsection{Datasets}

We built three test sets based on three samples of questions labelled with information intent that can be answered using unstructured text. Questions from Sample 1 are extracted from NQ questions while questions for samples 2 and 3 are generated from Alexa users' questions. For each questions, we selected 100 sentence candidates from the top documents retrieved by a search engine: (i) for samples 1 and 2, we used an elastic search system, ingested with several web domains, ranging from Wikipedia to reference.com, coolantarctica.com, www.cia.gov/library, etc. (ii) For Sample 3, we retrieved the candidate answers using a commercial search engine, to have a higher retrieval quality. 


\begin{tabular}{|c|c|c|c|c|c|c|c|c|c|c|c|}
\hline \multirow{2}{*}{\multicolumn{3}{|c|}{ MODEL }} & \multicolumn{3}{|c|}{ Sample 1 } & \multicolumn{3}{|c|}{ Sample 2} & \multicolumn{3}{|c|}{ Sample 3} \\
\hline & & & Prec@1 & MAP & MRR & Prec@1 & MAP & MRR & Prec@1 & MAP & MRR \\
\hline \multirow{6}{*}{ BERT } & \multirow{3}{*}{ Base } & NAD & 49.80 & 0.506 & 0.638 & 52.69 & 0.432 & 0.629 & 41.86 & 0.352 & 0.543 \\
\hline & & ASNQ & 55.06 & 0.557 & 0.677 & 44.31 & 0.395 & 0.567 & 44.19 & 0.369 & 0.561 \\
\hline & & TANDA (ASNQ $\rightarrow$ NAD) & 58.70 & 0.585 & 0.703 & 58.68 & 0.474 & 0.683 & 49.42 & 0.391 & 0.613 \\
\hline & \multirow{3}{*}{ Large } & NAD & 53.85 & 0.537 & 0.671 & 53.29 & 0.469 & 0.629 & 43.61 & 0.395 & 0.558 \\
\hline & & ASNQ & 57.49 & 0.552 & 0.686 & 50.89 & 0.440 & 0.630 & 45.93 & 0.399 & 0.585 \\
\hline & & TANDA (ASNQ $\rightarrow$ NAD) & 61.54 & 0.607 & 0.725 & 63.47 & 0.514 & 0.727 & 51.16 & 0.439 & 0.616 \\
\hline \multirow{6}{*}{ RoBERTa } & \multirow{3}{*}{ Base } & NAD & 59.11 & 0.563 & 0.699 & 56.29 & 0.511 & 0.670 & 48.26 & 0.430 & 0.612 \\
\hline & & ASNQ & 58.70 & 0.587 & 0.707 & 54.50 & 0.473 & 0.656 & 45.35 & 0.437 & 0.608 \\
\hline & & TANDA $($ ASNQ $\rightarrow$ NAD) & 65.59 & 0.623 & 0.757 & 62.87 & 0.537 & 0.714 & 56.98 & 0.473 & 0.679 \\
\hline & \multirow{3}{*}{ Large } & NAD & 70.81 & 0.654 & 0.796 & 63.47 & 0.581 & 0.734 & 52.91 & 0.490 & 0.651 \\
\hline & & ASNQ & 64.37 & 0.627 & 0.750 & 59.88 & 0.526 & 0.705 & 54.65 & 0.478 & 0.674 \\
\hline & & TANDA (ASNQ $\rightarrow$ NAD) & 71.26 & 0.680 & 0.805 & 74.85 & 0.625 & 0.821 & 58.14 & 0.514 & 0.699 \\
\hline
\end{tabular}

Table 9: Comparison between FT and TANDA on real-world datasets derived from Alexa Virtual Assistant traffic

The statistics of the three sample test sets are reported in Table 8. Their aim is to capture variations in terms of question sources and retrieval systems, thus providing more general results. Additionally, since questions without annotated answers do not affect system accuracy, the results we provide refer to a Clean setting, i.e., questions with all positive or negative answers are removed (no all+ and no all-).

\begin{tabular}{|c|c|c|c|c|}
\hline Data Split & Questions & QA Pairs & Neg. & Pos. \\
\hline Train & 25,226 & 134,765 & 125,779 & 8,986 \\
\hline Dev & 2,802 & 14,974 & 14,014 & 960 \\
\hline
\end{tabular}

Table 10: Statistics of the Noisy Alexa dataset (NAD)

We also built a noisy dataset (NAD) with a similar approach to Sample 2, with the restriction of retrieving only 10 candidates per question. This enables the cheaper annotation of a larger number of questions, which is important to build an effective training set. Indeed, the number of questions in NAD is one order of magnitude larger than those used for the previous samples (test questions). Additionally, NAD required an increased velocity of annotations resulting in a higher error rate, which we quantify around $20-25 \%$ (as estimated on a small sample). The statistics of NAD are presented in Table 10.

\subsection{Results}

In these experiments, we used, as usual, ASNQ for the transfer step, and NAD as our target dataset for the adapt step. Table 9 reports the comparative results using simple FT on NAD (denoted simply by NAD) and tested on samples 1, 2 and 3. We note that:

- applying ASNQ for the transfer step always provides improvement over FT.

- BERT Large TANDA improves over BERT Base TANDA for all the three different dataset samples.

- Using TANDA with RoBERTa produces an even higher improvement than with BERT.

- All these experiments using NAD for training and accurate datasets for testing, show that TANDA is robust to real-world noise of NAD as it always provides significantly large gains over simple FT.

\section{Conclusions}

In this paper, we have presented a novel approach for finetuning pre-trained Transformer models and tested it on a general natural language inference task, namely, answer sentence selection (AS2). Our approach, TANDA, performs two fine-tuning steps sequentially: (i) on a general, large and high-quality dataset, which transfers a pre-trained model to the target task; and (ii) on the target dataset to perform domain adaptation. The results on two well-known AS2 datasets, WikiQA and TREC-QA, show an impressive improvement over the state of the art. Additionally, our experiments in an industrial setting derive the same results and conclusions we found with the academic benchmarks.

Our research deepens the general understanding of transfer learning for Transformer models. The first step of TANDA produces an intermediate model with three main features: (i) it can be more effectively used for fine-tuning on the target NLP application, being more stable and easier to adapt to other tasks; (ii) it is robust to noise, which might affect the target domain data; and (iii) it enables modularity and efficiency, i.e., once a Transformer model is adapted to the target general task, e.g., AS2, only the adapt step is needed for each targeted domain. This is an important advantage in terms of scalability as the data of (possibly many) different target domains can be typically smaller than the dataset for the transfer step(ASNQ), thereby causing the main computation to be factorized on the initial transfer step.

We conjecture that one caveat of using simple fine-tuning on a combination of ASNQ and target data may produce an accuracy improvement similar to TANDA. However, such a combination can be tricky to optimize as the target data requires greater weighting than the more general data of ASNQ during fine-tuning the model. Our experiments with TREC-QA show that a simple union of the dataset with ASNQ is sub-optimal than sequential fine-tuning over ASNQ followed by TREC-QA. In any case, the important modular aspect of TANDA will not hold in such a scenario.

Interesting future work can be devoted to address the question about the applicability and generalization of the 
TANDA approach to other NLP tasks. In the specific context of AS2, it would be interesting to test if ASNQ can produce the same benefits for related but clearly different tasks, e.g., paraphrasing or textual entailment, where the relation between the members of text pairs are often different from those occurring between questions and answers.

\section{References}

Bian, W.; Li, S.; Yang, Z.; Chen, G.; and Lin, Z. 2017. A compareaggregate model with dynamic-clip attention for answer selection. In CIKM 2017, CIKM '17, 1987-1990. New York, NY, USA: ACM.

Chen, D.; Fisch, A.; Weston, J.; and Bordes, A. 2017. Reading wikipedia to answer open-domain questions. CoRR abs/1704.00051.

Dai, Z.; Yang, Z.; Yang, Y.; Carbonell, J. G.; Le, Q. V.; and Salakhutdinov, R. 2019. Transformer-xl: Attentive language models beyond a fixed-length context. CoRR abs/1901.02860.

Devlin, J.; Chang, M.; Lee, K.; and Toutanova, K. 2018. BERT: pre-training of deep bidirectional transformers for language understanding. CoRR abs/1810.04805.

He, H., and Lin, J. 2016. Pairwise word interaction modeling with deep neural networks for semantic similarity measurement. In NAACL 2016: Human Language Technologies, 937-948. San Diego, California: Association for Computational Linguistics.

Kingma, D. P., and Ba, J. 2014. Adam: A method for stochastic optimization. CoRR abs/1412.6980.

Kwiatkowski, T.; Palomaki, J.; Redfield, O.; Collins, M.; Parikh, A.; Alberti, C.; Epstein, D.; Polosukhin, I.; Kelcey, M.; Devlin, J.; Lee, K.; Toutanova, K. N.; Jones, L.; Chang, M.-W.; Dai, A.; Uszkoreit, J.; Le, Q.; and Petrov, S. 2019. Natural questions: a benchmark for question answering research. TACL.

Liu, Y.; Ott, M.; Goyal, N.; Du, J.; Joshi, M.; Chen, D.; Levy, O.; Lewis, M.; Zettlemoyer, L.; and Stoyanov, V. 2019. Roberta: A robustly optimized BERT pretraining approach. CoRR abs/1907.11692.

Luong, M.-T., and Manning, C. D. 2016. Achieving open vocabulary neural machine translation with hybrid word-character models. In Association for Computational Linguistics (ACL).

Min, S.; Seo, M.; and Hajishirzi, H. 2017. Question answering through transfer learning from large fine-grained supervision data. In ACL 2017, 510-517. Vancouver, Canada: Association for Computational Linguistics.

Peters, M. E.; Neumann, M.; Iyyer, M.; Gardner, M.; Clark, C.; Lee, K.; and Zettlemoyer, L. 2018. Deep contextualized word representations. CoRR abs/1802.05365.

Radford, A.; Wu, J.; Child, R.; Luan, D.; Amodei, D.; and Sutskever, I. 2018. Language models are unsupervised multitask learners.

Rajpurkar, P.; Zhang, J.; Lopyrev, K.; and Liang, P. 2016. Squad: $100,000+$ questions for machine comprehension of text. CoRR $\mathrm{abs} / 1606.05250$.

Seo, M. J.; Kembhavi, A.; Farhadi, A.; and Hajishirzi, H. 2016. Bidirectional attention flow for machine comprehension. CoRR $\mathrm{abs} / 1611.01603$.

Severyn, A., and Moschitti, A. 2015. Learning to rank short text pairs with convolutional deep neural networks. In Proceedings of the 38th International ACM SIGIR Conference on Research and Development in Information Retrieval, SIGIR '15, 373-382. New York, NY, USA: ACM.
Severyn, A., and Moschitti, A. 2016. Modeling relational information in question-answer pairs with convolutional neural networks. CoRR abs/1604.01178.

Shen, G.; Yang, Y.; and Deng, Z.-H. 2017. Inter-weighted alignment network for sentence pair modeling. In EMNLP 2017, 11791189. Copenhagen, Denmark: Association for Computational Linguistics.

Sun, C.; Qiu, X.; Xu, Y.; and Huang, X. 2019. How to fine-tune BERT for text classification? CoRR abs/1905.05583.

Tay, Y.; Tuan, L. A.; and Hui, S. C. 2018. Multi-cast attention networks for retrieval-based question answering and response prediction. CoRR abs/1806.00778.

Tayyar Madabushi, H.; Lee, M.; and Barnden, J. 2018. Integrating question classification and deep learning for improved answer selection. In Proceedings of the 27th International Conference on Computational Linguistics, 3283-3294. Santa Fe, New Mexico, USA: Association for Computational Linguistics.

Tran, Q. H.; Lai, T.; Haffari, G.; Zukerman, I.; Bui, T.; and Bui, H. 2018. The context-dependent additive recurrent neural net. In NAACL 2018: Human Language Technologies, Volume 1 (Long Papers), 1274-1283. New Orleans, Louisiana: Association for Computational Linguistics.

Vaswani, A.; Shazeer, N.; Parmar, N.; Uszkoreit, J.; Jones, L.; Gomez, A. N.; Kaiser, L. u.; and Polosukhin, I. 2017. Attention is all you need. In Advances in Neural Information Processing Systems 30. Curran Associates, Inc. 5998-6008.

Wang, S., and Jiang, J. 2016. A compare-aggregate model for matching text sequences. CoRR abs/1611.01747.

Wang, A.; Singh, A.; Michael, J.; Hill, F.; Levy, O.; and Bowman, S. R. 2018. GLUE: A multi-task benchmark and analysis platform for natural language understanding. CoRR abs/1804.07461.

Wang, R.; Su, H.; Wang, C.; Ji, K.; and Ding, J. 2019. To tune or not to tune? how about the best of both worlds? ArXiv abs/1907.05338.

Wang, M.; Smith, N. A.; and Mitamura, T. 2007. What is the Jeopardy model? a quasi-synchronous grammar for QA. In (EMNLPCoNLL) 2007, 22-32. Prague, Czech Republic: Association for Computational Linguistics.

Yang, L.; Ai, Q.; Guo, J.; and Croft, W. B. 2018. anmm: Ranking short answer texts with attention-based neural matching model. CoRR abs/1801.01641.

Yang, W.; Xie, Y.; Lin, A.; Li, X.; Tan, L.; Xiong, K.; Li, M.; and Lin, J. 2019. End-to-end open-domain question answering with BERTserini. In NAACL 2019, 72-77. Minneapolis, Minnesota: Association for Computational Linguistics.

Yang, Y.; Yih, W.-t.; and Meek, C. 2015. WikiQA: A challenge dataset for open-domain question answering. In EMNLP 2015, 2013-2018. Lisbon, Portugal: Association for Computational Linguistics.

Yoon, S.; Dernoncourt, F.; Kim, D. S.; Bui, T.; and Jung, K. 2019. A compare-aggregate model with latent clustering for answer selection. CoRR abs/1905.12897. 